\title{
Charge and colour breaking bounds revisited
}

\author{
P.M. Ferreira \\ Istituto Nazionale di Fisica Nucleare, \\ Sezione di Genova, Italia
}

ABstRACT: Charge and colour breaking bounds in supersymmetric models are re-analyzed, with special attention given to the importance of the 1-loop corrections to the tree-level potential. It is argued that the usual way of determining these bounds over estimates their relevance, and a new method is proposed and applied to the MSSM with universality.

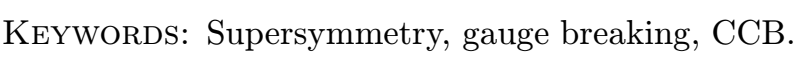

\section{Introduction}

In the Standard Model (SM), the Higgs field acquires a non-zero vev which, this field being an $S U(2)$ doublet and having hypercharge $+1 / 2$, breaks down the $S U(2)_{L} \times U(1)_{Y}$ symmetry. Because the Higgs boson is chargeless and colourless, the gauge group $S U(3)_{C} \times U(1)_{e m}$ remains unbroken. No other fields acquire vevs - the Higgs is the only scalar field in the model, and if a field with non-zero spin were to acquire a vev angular momentum would not be conserved. In supersymmetric models, in order to give mass both to the up and down-type quarks (and also to cancel chiral and $S U(2)$ anomalies), two Higgs doublets, $H_{1}$ and $H_{2}$, are required. The neutral components of these fields have a non-zero vev which, much in the same manner as in the SM, breaks the electroweak gauge symmetry. However, there are now many other scalar fields in the game, many of them carrying charge, colour or leptonic number, and a priori there is no reason why one or many of them cannot have a vev. This problem was first addressed in ref. [i]1], and used to impose restrictions on the undesirably large parameter space of supersymmetric models. This was done by demanding that the minimum of the potential along CCB directions (meaning, when fields other than $H_{1}^{0}$ and $H_{2}^{0}$ acquire vevs) be less deep than the so-called "real" minimum. This implied, for a trilinear term in the potential of the form $\lambda A X Y Z$ (where $\lambda$ is an Yukawa coupling, $A$ the associated trilinear soft super- symmetry breaking term and $X, Y$ and $Z$ generic fields), the famous bounds on the $A$-parameters,

$$
A^{2}<3\left(m_{X}^{2}+m_{Y}^{2}+m_{Z}^{2}\right)
$$

where $m_{X}, m_{Y}$ and $m_{Z}$ are the soft masses of the corresponding fields. This very appealing idea was developed by many authors $\left[\begin{array}{lll}\overline{2}+1 & \overline{1} \\ -1 & 3\end{array}\right]$, and of particular importance was the contribution from Gamberini et al [4]: they showed that the oneloop corrections to the effective potential in supersymmetric theories were of immense relevance for gauge symmetry breaking, in that only at a given renormalization scale $\mu$ - namely, $\mu$ of the order of the most significant mass appearing in the one-loop corrections - can their contribution be neglected and results derived from the treelevel potential trusted. Given that every work on CCB is based on an analysis of the tree-level potential only, they concluded that any bound on supersymmetric parameters we obtain in this way, if not evaluated at the scale $\mu$, will lead to an excessive constriction on the parameter space [in] Recent use of CCB bounds in supersymmetric phenomenology may be found in ref. [i,

\section{Usual CCB bounds}

The typical procedure to find a CCB bound for a given theory consists of expressing all vevs involved in terms of one of them, say, $v_{2}$, the vev of $H_{2}$, so that $v_{1}=\alpha v_{2}, q=\beta v_{2}, \ldots$, where $v_{1}$ is the vev of $H_{1}$ and $q$ that of some field $Q$ carrying 
colour. The potential $V\left(v_{2}, \alpha, \beta, \ldots\right)$ is then minimised with respect to $v_{2}$, keeping the remaining parameters fixed. Finding the value of the potential at this minimum, we can them compare it with the "real" minimum - meaning, the value of the potential when the only vevs present are $v_{1}$ and $v_{2}$ - and require the latter to be smaller. In many cases an analytical analysis is possible and one finds a condition of the form

$$
f(\mathbf{A})<H(\alpha, \beta, \ldots),
$$

where $f$ is some function of the parameters $\mathbf{A}$ (usually only the trilinear couplings $A$ ). The CCB bound is found by minimising $H$ with respect to $\alpha, \beta, \ldots$. The only restrictions upon these parameters are that they are positive (involving a previous choice for the phases of the vevs) and such that the minimum of $V$, as found by solving $\partial V / \partial v_{2}=0$, exists.

The superpotential of the MSSM, keeping only the Yukawa couplings for the third generation, is given by

$$
\begin{aligned}
W= & \lambda_{t} H_{2} Q t_{R}+\lambda_{b} H_{1} Q b_{R}+\lambda_{\tau} H_{1} L \tau_{R} \\
& +\mu H_{1} H_{2}
\end{aligned}
$$

where $Q=\left(\begin{array}{ll}t_{L} & b_{L}\end{array}\right)$ and $L=\left(\begin{array}{ll}\nu_{L} & \tau_{L}\end{array}\right)$ are the quark and lepton $S U(2)_{L}$ doublet superfields, respectively. $t_{R}, b_{R}$ and $\tau_{R}$ are singlets of the same gauge group, the $\lambda_{i}$ are the Yukawa couplings and $\mu$ is the well-known bilinear coupling.

When the neutral components of $H_{1}$ and $H_{2}$ develop vevs $v_{1}$ and $v_{2}$, we obtain the "real" minimum, and the potential $V_{0}$ may be written as

$$
\begin{aligned}
V_{0}= & m_{1}^{2}\left|v_{1}\right|^{2}+m_{2}^{2}\left|v_{2}\right|^{2}-2\left|m_{3}^{2}\right|\left|v_{1} v_{2}\right| \\
& +\frac{1}{8}\left(g^{\prime 2}+g_{2}^{2}\right)\left(\left|v_{2}\right|^{2}-\left|v_{1}\right|^{2}\right)^{2},
\end{aligned}
$$

where as usual $m_{1}^{2}=m_{H_{1}}^{2}+\mu^{2}, m_{2}^{2}=m_{H_{2}}^{2}+\mu^{2}$ and $m_{3}^{2}=-B \mu$, and we chose the phases of the vevs so that the $m_{3}^{2}$ term is negative. It is a trivial matter to minimise this expression with respect to $v_{1}$ and $v_{2}$ and obtain the value of the potential at the "real" minimum,

$$
V_{\text {min }}=-\frac{1}{8}\left(g^{\prime 2}+g_{2}^{2}\right)\left(\left|v_{2}\right|^{2}-\left|v_{1}\right|^{2}\right)^{2} .
$$

We left out the one-loop corrections to the scalar potential, given by

$$
V_{1}=\sum_{\alpha} \frac{n_{\alpha}}{64 \pi^{2}} M_{\alpha}^{4}\left(\log \frac{M_{\alpha}^{2}}{\mu^{2}}-\frac{3}{2}\right),
$$

where the $M_{\alpha}$ are the (tree-level) masses of each particle of spin $s_{\alpha}$ and $n_{\alpha}=(-1)^{2 s_{\alpha}}\left(2 s_{\alpha}+1\right)$. As first shown in ref. [4ind, if we choose the scale $\mu$ to be of order of the largest mass $M_{\alpha}$, these one-loop corrections will be quite small and results derived from the tree-level potential can be trusted.

When looking for CCB minima, one tries to select the cases which will in principle produce smaller values of the potential: meaning, those in which the negative terms in the potential are large and the positive, quartic $\mathrm{F}$ and D-terms are small. An excellent review on the subject may be found in ref. their results to emphasise the differences in our approach. Essentially, any CCB minimum will have to involve an extra negative term other than the $m_{3}^{2}$ one present in eq. to the soft breaking trilinear terms. For such terms to have a contribution to $V_{0}$, at least two fields other than $H_{1}$ and $H_{2}$ must acquire a vev $Q$ and $t_{R}$ in the case of the top trilinear term, for example. So that that contribution is negative, the phases of the vevs must be chosen accordingly. This is not always possible, but for the moment we will just assume it. Of course, as new vevs enter the game, new positive contributions appear - from the scalar masses, from F-terms involving Yukawa couplings, from additional contributions to the D-terms. These last ones make it particularly difficult an analytical handling of this subject. The $S U(3)$ D-term, due to the size of the strong coupling, is almost always set to zero by choosing a CCB direction along which the $Q$ doublet (and colour triplet) and one of the $t_{R}, b_{R}$ singlets (and colour triplets) have identical vevs, an assumption that considerably simplifies calculations. The electroweak D-terms may be reduced in size if a lepton doublet $L$ gains a vev - this possibility is quite appealing, in that it does not introduce extra F-terms. Of course, a vev for $L$ introduces an extra soft mass term in the potential, so $L$ should be the doublet from the third generation, which has the lightest soft mass. With a vev for $Q, t_{R}, H_{1}, H_{2}$ and $L$, the scalar potential is given by

$$
\begin{aligned}
V_{0}= & \lambda_{t}^{2}\left(q^{4}+2 q^{2} v_{2}^{2}\right)-2 \lambda_{t} q^{2}\left(A_{t} v_{2}+\mu v_{1}\right) \\
& +m_{1}^{2} v_{1}^{2}+m_{2}^{2} v_{2}^{2}-2\left|m_{3}^{2}\right| v_{1} v_{2}
\end{aligned}
$$




$$
\begin{aligned}
& +\left(m_{Q}^{2}+m_{t_{R}}^{2}\right) q^{2}+m_{L}^{2} l^{2} \\
& +\frac{1}{8}\left(g^{\prime 2}+g_{2}^{2}\right)\left(v_{2}^{2}-v_{1}^{2}-q^{2}-l^{2}\right)^{2}
\end{aligned}
$$

where for simplicity of writing $q$ is the modulus of the vev of both $Q$ and $t_{R}, v_{1}, v_{2}$ and $l$ are the modulus of the vevs of $H_{1}, H_{2}$ and $L$ respectively and $A_{t}, \mu$ are the modulus of the trilinear and bilinear couplings. So that no undesirable quartic F-terms are produced, $Q$ has a vev along the $t_{L}$ direction and $L$ along the $\nu_{L}$ direction. In

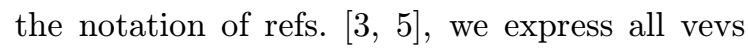
in terms of $v_{2}$, so that $q=\alpha v_{2}, v_{1}=\beta v_{2}$ and $l=\gamma v_{2}$. The potential $\underline{2}-\overline{6}$ becomes a quartic polynomial in $v_{2}$ and its minimisation a trivial exercise. $\partial V_{0} / \partial v_{2}=0$ produces

$$
v_{2_{m i n}}=\frac{3 \hat{A}}{4 \lambda_{t} \alpha^{2} F}\left(1+\sqrt{1-\frac{8 \hat{m}^{2} F}{9 \hat{A}^{2}}}\right),
$$

where

$$
\begin{aligned}
F= & \frac{\left(g^{\prime 2}+g_{2}^{2}\right)}{8 \lambda_{t}^{2} \alpha^{4}}\left(1-\alpha^{2}-\beta^{2}-\gamma^{2}\right)^{2} \\
& +\frac{2}{\alpha^{2}}+1, \\
\hat{A}= & A_{t}+\mu \beta, \\
\hat{m}^{2}= & m_{2}^{2}+\left(m_{Q}^{2}+m_{t_{R}}^{2}\right) \alpha^{2}+m_{1}^{2} \beta^{2} \\
& +m_{L}^{2} \gamma^{2}-2\left|m_{3}^{2}\right| \beta .
\end{aligned}
$$

The existence of the solution given by eq. constrains the parameters $\alpha, \beta$ and $\gamma$. The value of the potential at this minimum is then given by

$$
V_{m i n}^{C C B}=-\frac{1}{2} v_{2_{m i n}}^{2}\left(\alpha^{2} \lambda_{t} \hat{A} v_{2_{m i n}}-\hat{m}^{2}\right)
$$

The CCB bounds are then found by comparing this with the "real" minimum of eq. 2.4 . Because vevs related to a given trilinear term of coupling $\lambda$ and coefficient $A$ are expected to be of order

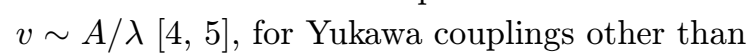
the top's the potential of eq. 2.9 is expected, if negative, to be much deeper than that of eq. so that the $\mathrm{CCB}$ bound is found in a simpler way: by requiring that $V_{\min }^{C C B}$ be positive, that is, $\hat{A}^{2} \leq F \hat{m}^{2}$. A simple such example is to consider a coupling $\lambda_{u}$ (from an up-quark of the second or first generations), the vanishing of the D-terms enforced by choosing the $L$ vev such that $\gamma=$ $1-\alpha^{2}-\beta^{2}$. If we consider an even simpler case that the field $H_{1}$ acquires no vev - and set $\beta=0$, the CCB condition becomes quite simple,

$$
\begin{aligned}
A_{u}^{2} \leq & \left(1+\frac{2}{\alpha^{2}}\right)\left[m_{2}^{2}+m_{L}^{2}\right. \\
& \left.+\left(m_{Q_{u}}^{2}+m_{u}^{2}-m_{L}^{2}\right) \alpha^{2}\right]
\end{aligned}
$$

with the obvious substitutions $t \rightarrow u$. Then, according to the authors of [is] constraint is found by minimising the right hand side of this equation with respect to $\alpha$. In the case where $m_{2}^{2}+m_{L}^{2}>0$ and $2\left(m_{2}^{2}+m_{L}^{2}\right)>$ $m_{Q_{u}}^{2}+m_{u}^{2}-m_{L}^{2}, \alpha=1$ is obtained and the "classical" bound from eq.

$$
A_{u}^{2} \leq 3\left(m_{2}^{2}+m_{Q_{u}}^{2}+m_{u}^{2}\right)
$$

\section{Objections to the usual CCB pro- cedure}

At this point, we ask: how can we be sure that there $i s$ a minimum of $V_{0}$ such that $\alpha=1$ (or better put, $q=v_{2}$ ) when the only minimisation we performed on the potential was that with respect to $v_{2}$ ? Given that the minimum of $V_{0}$ should be found by solving the equations $\partial V_{0} / \partial v_{n}=0$ where the derivatives are performed with respect to each vev present in $V_{0}$-, not all values of $q$, $v_{1}, l$ and $v_{2}$ will be solutions. It seems obvious that the solution space of this set of equations is smaller than that which is found by requiring that the potential of eq. $2 \cdot \overline{9}$ exists. Thus the minimisation of the right-hand side of eq. 'i2.10 should be made for a more restrict range of values of $\alpha$. On the other hand, as they arise from a $n$-variable system of $n$ equations, the values of the vevs at the minimum will be closely correlated. What that correlation is, can only by found by solving the afore-mentioned equations, but we certainly cannot guarantee it is simply linear as has been assumed until now. Further, though the use of the parameters $\alpha, \beta, \ldots$ considerably simplifies the study of the CCB minima, it is not clear that solving $\partial V_{0}\left(v_{2}, \alpha, \beta, \ldots\right) / \partial v_{2}=0$ is the same as solving $\partial V_{0}\left(v_{2}, v_{1}, q, \ldots\right) / \partial v_{2}=0$ - in the first case, the potential becomes a quartic polynomial on $v_{2}$ where the lowest term is quadratic. The value of $v_{2}$ that arises from the minimisation is the result of solving a quadratic 
equation. In the second case, as we will shortly show, $v_{2}$ is far more difficult to determine, but in most cases it is the solution of a cubic equation. That these two values of $v_{2}$ should match is not at all obvious.

The main objection, though, relates to the 1-loop corrections to the potential - as is plain from eq. 2.5 , these corrections are defined at a minimum of the tree-level potential, given the fact that they boast logarithms whose arguments are the tree-level mass matrices' eigenvalues. To be more accurate, these corrections are derived under the assumption that the potential is resting at a tree level minimum. The issue of complex effective potentials has been the subject of much study [7] and understood to arise when nonconvex classical potentials are present. In short, these complex contributions are a signal that the sum of one-particle irreducible diagrams, $V_{1 P I}$, precisely in the case of nonconvex potentials, does not give the effective potential $V_{\text {eff }}$ in those cases, $V_{\text {eff }}$ is given by the convex envelope of $V_{1 P I}$ which, in the vicinity of the classical minimum, matches the usual loop expansion. Now, following the CCB procedure detailed above, one is not guaranteed to actually find minima, only "points" of parameter space in which the tree-level potential - evaluated at a given scale for which one expects the 1-loop contributions to the potential to be negligible is smaller than the non-CCB minimum. We will show in a simple example that the 1-loop contributions to the potential are actually quite sizeable, even if one chooses an energy scale of the order of the largest mass in eq.

\section{An example: vevs for $v_{1}, \tau_{L}, \tau_{R}$.}

In this section we will illustrate the arguments of the previous one and study the effective potential in a direction which is not a minimum but for which one finds CCB. In anticipation of the next section, we will only consider "points" of parameter space for which the minimisation conditions can be solved. Due to the bilinear term in $H_{1}$ and $H_{2}$, for a potential like that of eq. 2.6 any direction characterised by $v_{2}=0$ and $v_{1} \neq 0$ is guaranteed not to be a minimum of the potential. But we shall find that, considering non-zero vevs for $v_{1}, \tau_{L}$ and $\tau_{R}$, one finds, at least for some regions of parameter space, that the CCB potential is smaller in value than the "real" minimum. There is no colour breaking in this case, of course, but we will still use the abbreviation CCB as an abuse of language. The tree-level potential along this direction is given by

$$
\begin{aligned}
V_{0}= & \lambda_{\tau}^{2}\left(l^{4}+2 l^{2} v_{1}^{2}\right)-2 \lambda_{\tau} l^{2} A_{\tau} v_{1} \\
& +m_{1}^{2} v_{1}^{2}+\left(m_{L}^{2}+m_{\tau_{R}}^{2}\right) l^{2} \\
& +\frac{1}{8}\left(g^{\prime 2}+g_{2}^{2}\right)\left(l^{2}-v_{1}^{2}\right)^{2}
\end{aligned}
$$

and we are considering a direction along which the vevs for $\tau_{L}$ and $\tau_{R}$ are both equal to $l$ (allowing these two vevs to differ doesn't actually change the results obtained). The phases of the vevs and signal of couplings were chosen so that the trilinear term in the previous equation is negative, and all quantities figuring there are therefore positive. Minimising with respect to $v_{1}$ and $l$, we find that $l^{2}$ is determined from

$$
\begin{aligned}
{\left[\frac{1}{8}\left(g^{\prime 2}+g_{2}^{2}\right)+\right.} & \left.\lambda_{\tau}^{2}\right] l^{2} \\
= & {\left[\frac{1}{8}\left(g^{\prime 2}+g_{2}^{2}\right)-\lambda_{\tau}^{2}\right] v_{1}^{2} } \\
& +\lambda_{\tau} A_{\tau} v_{1} \\
& -\frac{1}{2}\left(m_{L}^{2}+m_{\tau_{R}}^{2}\right)
\end{aligned}
$$

and $v_{1}$ is the solution of the cubic equation

$$
\begin{aligned}
0= & 2 \lambda_{\tau}^{2}\left[\frac{3}{8}\left(g^{\prime 2}+g_{2}^{2}\right)-\lambda_{\tau}^{2}\right] v_{1}^{3} \\
& -3 \lambda_{\tau}\left[\frac{1}{8}\left(g^{\prime 2}+g_{2}^{2}\right)-\lambda_{\tau}^{2}\right] A_{\tau} v_{1}^{2} \\
& +\left[\lambda_{\tau}^{2}\left(m_{1}^{2}-A_{\tau}^{2}-m_{L}^{2}-m_{\tau_{R}}^{2}\right)\right. \\
& \left.+\frac{1}{8}\left(g^{\prime 2}+g_{2}^{2}\right)\left(m_{1}^{2}+m_{L}^{2}+m_{\tau_{R}}^{2}\right)\right] v_{1} \\
& +\frac{1}{2} A_{\tau}\left(m_{L}^{2}+m_{\tau_{R}}^{2}\right) .
\end{aligned}
$$

The procedure we follow is quite simple: solve this equation for the value of $v_{1}$ - remember $v_{1}$ is supposed to be positive (or at least real - a negative $v_{1}$ would correspond to a positive value of $V_{0}$ and therefore be uninteresting for CCB), thus determining $l^{2}$ from it. A negative value for $l^{2}$ would mean that it is impossible to solve the minimisation equations along this direction. 


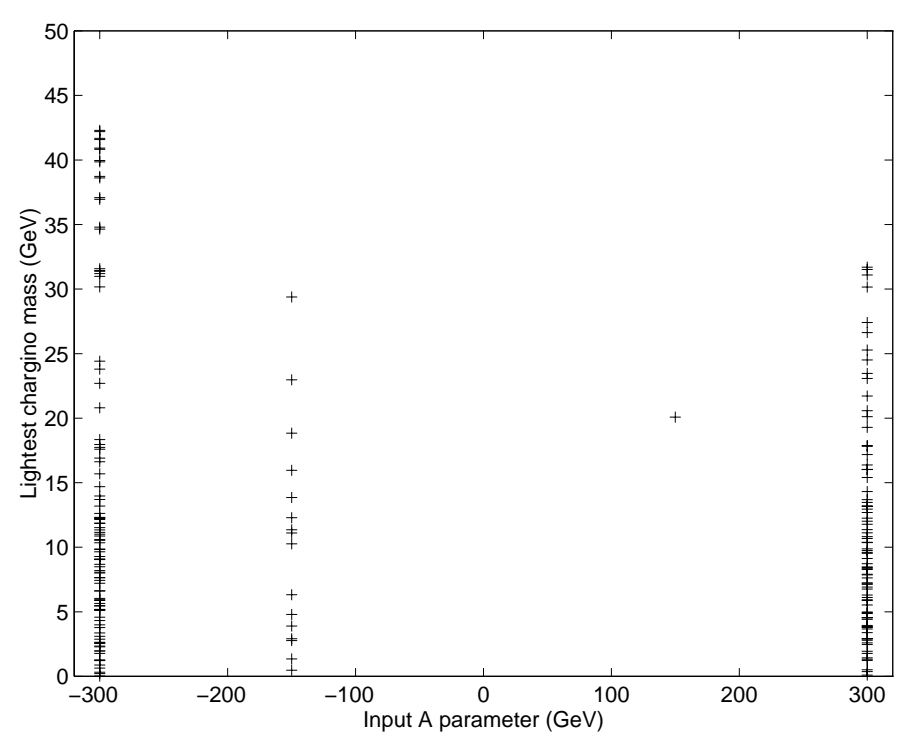

Figure 1: Input $A$ parameter vs. lightest chargino mass for the CCB excluded region.

When a minimum along this direction is found, the value of the potential is compared to that of the "real" minimum and, if smaller, the "point" of parameter space under consideration is singled out as possibly dangerous for CCB. As usual, we choose to work at a renormalization scale of the order of the largest masses induced in the potential by the non-zero vevs. We did compute those masses, but an equally effective choice is to take $\mu^{2}$ of the order of the largest of the second derivatives of the potential in eq. 4 with respect to $l$ and $v_{1}$.

We applied this procedure to a simple example - the MSSM with universality, in the "low mass" region described in the next section (values of the gaugino mass between 10 and $100 \mathrm{GeV}$, values of the universal $A$ parameter between -300 and $300 \mathrm{GeV}$ ). Out of a parameter space with 850 "points", we found out about 200 CCB minima along this direction. CCB seems to occur for large absolute values of the $A$ parameter, as can be seen from figure $v_{1}$ and $l$ are very close - in hindsight this was to be expected, as it causes the vanishing of the Dterms in eq. 4.1. Also, these vevs, as one expects from equation 4.3 , are of order $\mathcal{M} / \lambda_{\tau}-\mathcal{M}$ being a mass scale of the order of the several soft parameters from which $v_{1}$ is determined - which in this case corresponds roughly to a scale of several
TeV. Notice how these CCB "points" correspond to values of the mass of the lightest chargino always inferior to $45 \mathrm{GeV}$ - that is, inferior to the current experimental bound on that variable [is]

Now, these results arise from calculations of the tree level potential, at an energy scale for which one argues the 1-loop contributions to the potential are negligible. We tested this assumption, by computing those contributions, which involved the calculation of the mass matrices of all the particles of the theory ${ }^{1}$. As expected since we are not at a minimum of the potential, several of the eigenvalues of these matrices are negative - largely so, in fact - and we are thus left with a complex effective potential. We know from refs. [i]|] that this is the result of a breakdown of the usual loop expansion for the effective potential, which is valid only in the vicinity of classical minima. At this point, we may assume two positions: one, more extreme, arguing that the results of refs. [i] seem to apply to cases where, despite non-convexity of the effective potential, one is still in the vicinity of minima. In the example we are studying the potential is not in a minimum, and in fact may be quite far from one (a $v_{2}=0$ minimum implies

\footnotetext{
${ }^{1}$ These are not, of course, the usual mass matrices of the MSSM, which can be found in references like $\left[\hat{9}^{4}\right]$, but those that arise when non-zero vevs for $H_{1}^{0}, \tau_{L}$ and $\tau_{R}$ are present.
} 


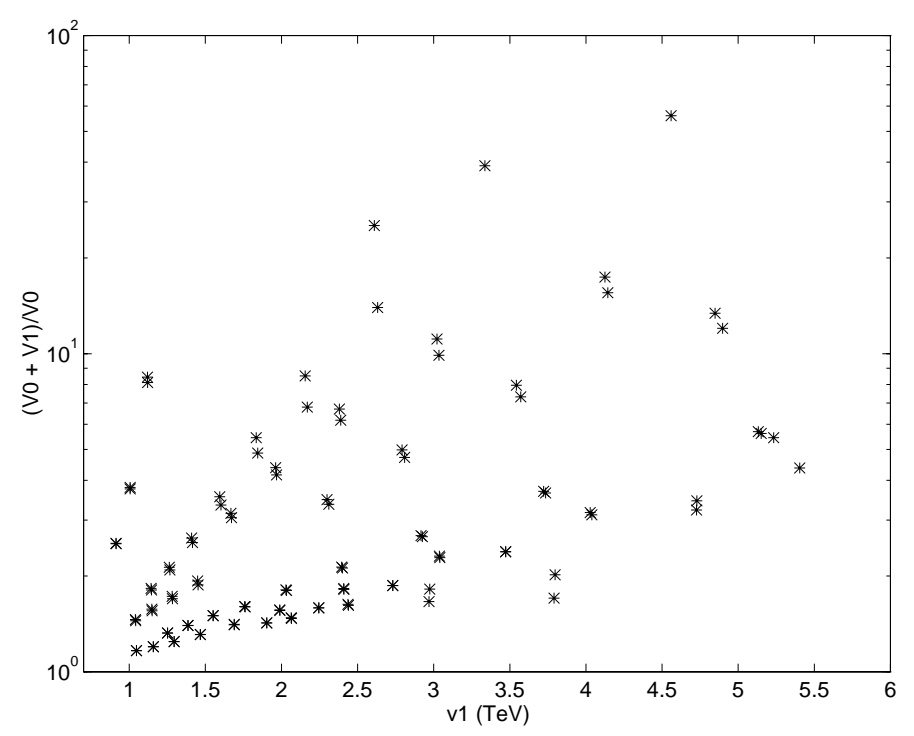

Figure 2: Value of $v_{1}$ versus the ratio $\left(V_{0}+V_{1}\right) / V_{0}$.

$v_{1}=0$, whereas we have values of $v_{1}$ of the $\mathrm{TeV}$ order). We therefore would have to accept that the 1-loop potential is simply not defined along this direction, which puts in question any results derived from $V_{0}$. A second position, more moderated, would consist of trying to estimate the 1-loop contributions to the effective potential assuming that, even along this direction, $V_{\text {eff }}$ is given by the real part of the 1-loop potential (a la Weinberg and $\mathrm{Wu}[\overline{1}]$ ) or by the convex envelope of the classical potential (Fujimoto et al [īi] - with so many fields involved, building the convex envelope is nigh impossible, but we believe a good estimation is obtained by simply setting to zero the contributions of the negative eigenvalues to $\left.V_{1}\right)$. Both approaches, however, produce similar results. In figure $\overline{2}_{1}^{1}$ we plot $v_{1}$ versus the ratio of the full one loop and the tree level potentials. By choosing the renormalization scale of the order of the largest masses present in $V_{1}$, we expected to make its contributions negligible, but we see that is not the case - for over half of the CCB "minima", $V_{1}$ is at least as large as $V_{0}$ and in many cases much larger. The conclusion? Even with this moderated viewpoint, the 1-loop contributions are too large and cast doubt over the results obtained from the tree level potential.

\section{Determining the vevs from the minimisation conditions}

To address these objections, we propose taking the (somewhat) brute-force approach: for a given CCB scenario, where $n$ fields gain vevs, solve the $n$ minimisation conditions and calculate the potential at the new minimum. If this is found to be smaller in value that the "real" minimum of eq. 2.4 , this "point" in the MSSM parameter space will be rejected. The undesirable feature of this approach is that it will not generate analytical expressions for the bounds such as eq. $\overline{2} \cdot \overline{1}_{1}$. Nevertheless, it produces remarkably simple equations (in most cases) for the vevs and the minimum of the potential, and seems a more correct approach to CCB - determining minima we are assured that the 1-loop contributions are defined and the correct choice of renormalization scale renders them small. But notice that we are going from solving a simple quadratic equation (that which determines $v_{2_{m i n}}$, eq. $\overline{2} . \overline{7}$ ) to solving a set of $n$ non-linear equations - we may expect that solving this set of equations is impossible for a great deal of the parameter space, and in fact this is what we will find shortly. For illustration, consider the potential of eq. $\overline{2} \cdot \overline{6}$. Minimising it with respect to the vev $l$, we find there are two 
solutions, $l=0$ and

$$
l^{2}=v_{2}^{2}-v_{1}^{2}-q^{2}-\frac{4 m_{L}^{2}}{g^{\prime 2}+g_{2}^{2}} .
$$

Notice how in this expression the only positive term is that of $v_{2}^{2}$, so that negative values of $l^{2}$ are likely. This is far from being the only such case - with $l=0$, and assuming an $S U(3)$ D-flat direction, $q^{2}$ is given by

$$
\begin{aligned}
& {\left[\frac{1}{8}\left(g^{\prime 2}+g_{2}^{2}\right)-\lambda_{t}^{2}\right] v_{2}^{2}-\frac{1}{8}\left(g^{\prime 2}+g_{2}^{2}\right) v_{1}^{2} } \\
& +\lambda_{t}\left(A_{t} v_{2}+\mu v_{1}\right)-\frac{1}{2}\left(m_{Q}^{2}+m_{t_{R}}^{2}\right) \\
= & {\left[\frac{1}{8}\left(g^{\prime 2}+g_{2}^{2}\right)+\lambda_{t}^{2}\right] q^{2} . }
\end{aligned}
$$

The large size of $\lambda_{t}$ will almost certainly drive the $v_{2}^{2}$ term negative. Also, the soft masses have a sizeable and negative contribution. $v_{1}$ and $v_{2}$ are the solutions of a set of two cubic equations and are determined numerically, and the potential at this extremum is given by the simple expression

$$
\begin{aligned}
V_{e x t}= & -\frac{1}{8}\left(g^{\prime 2}+g_{2}^{2}\right)\left(v_{2}^{2}-v_{1}^{2}\right)^{2} \\
& +q^{2}\left[\frac{1}{2}\left(m_{Q}^{2}+m_{t_{R}}^{2}\right)-\lambda_{t}^{2} v_{2}^{2}\right. \\
& \left.+\frac{1}{8}\left(g^{\prime 2}+g_{2}^{2}\right)\left(v_{2}^{2}-v_{1}^{2}\right)\right] .
\end{aligned}
$$

Again, the soft mass terms work against the possibility of CCB - even if it is possible to solve the minimisation conditions, these terms have a sizeable positive contribution to $V_{0}$.

We applied these considerations to a simple case, that of the MSSM with universality - that is, common values of the soft parameters $m_{i}, M_{i}$ and $A_{i}$ at the unification scale $M_{U}$. We considered two different regions of the MSSM parameter space. The first is a "low mass" region, characterised by small values for the gaugino mass. We took $M$ equal to $10,20,40,80$ and $100 \mathrm{GeV}$, $m$ equal to $10,20,40,80,160 \mathrm{GeV}, A$ equal to $-300,-150,0,150$ and $300 \mathrm{GeV}$, and values of $\tan \beta$ between 2.5 and 10.5 (smaller values of $\tan \beta$ cause problems with the running of the soft $\beta$-functions - the two-loop terms become more significant than the one-loop ones and Landau poles appear, in the running of $m_{Q}$, for example). The second region includes the same values for $\tan \beta$, values of $M$ from 50 to $300 \mathrm{GeV}$ in leaps of $50 \mathrm{GeV}$, values of $m$ from 50 to $500 \mathrm{GeV}$, also in leaps of $50 \mathrm{GeV}$, values of $A$ from -300 to $300 \mathrm{GeV}$, in leaps of $30 \mathrm{GeV}$. The first region includes about 850 "points", the second, some 15000 after we imposed experimental bounds on the sparticles' masses (as quoted in ref. [ipl). We analysed the cases of CCB breaking from the trilinear couplings involving the top, bottom and tau couplings, in a variety of cases - with and without $l$ vevs (to try reducing the size of the Dterms), accounting for the different possibilities in the choice of phases of the vevs, even considering some $S U(3)$ non-flat directions (for the case of the top, for instance, that consists in taking different vevs for $t_{L}$ and $t_{R}$; a large $S U(3)$ Dterm then appears).

Scanning all this parameter space, we found no dangerous CCB minima. For the majority of "points", solving the minimisation conditions reveals itself impossible - and when an extremum of the potential does exist, it is found to be larger than the "real" minimum of eq. 2.4 . This is, in great measure, due to the soft masses' contributions to the potential - they are large and positive. Of course, had we found any CCB minima, we would have to have taken into account the possibility of metastable vacua - that is, if the standard, non CCB vacuum is a metastable one with a lifetime larger than the current age of the universe, the new CCB minimum would not be dangerous [1]

\section{Discussion}

In these proceedings we touched the main features of this subject. More details may be found

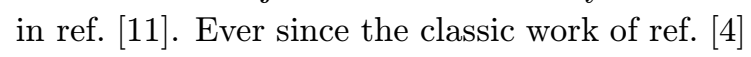
we know that failing to take into account the 1-loop corrections to the effective potential can lead to an overestimation of CCB bounds. Also, these corrections can have quite drastic effects on predictions from the tree-level potential. When studying CCB, one usually tries to minimise the 1-loop contributions by an appropriate choice of renormalization scale. But a global minimum of the potential is not searched, instead a strategy of finding regions of parameter space where the CCB tree-level potential has a value inferior to that of the "real" minimum is followed. The 
argument is that if that if that point is not a minimum, a valid minimum, or an unbounded from below (UFB) direction, exists surely somewhere. But as we discussed in section "it, away from a minimum, even with a judicious choice of renormalization scale, the 1-loop corrections are not guaranteed to be small, and indeed are not. And this, of course, if one ignores the rather uncomfortable fact that in such regions the 1loop contributions are complex - notice that this is a rather different situation from that found in ref. [i] $[\overline{4}]$, where a small displacement from the minimum caused the appearance of small negative squared masses for the Goldstone bosons. In our example, the negative squared masses we found were very sizeable.

We argued that the sensible way to follow is

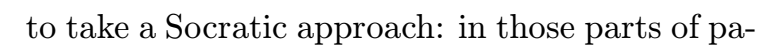
' rameter space_ where_a tree-level minimum cannot be found, the 1-loop corrections cannot be handled in the usual way and, even if the potential happens to be smaller than the "real" minimum, no CCB statements can be made. We applied this philosophy to the MSSM with univer- satity,-for a -wide range-of-paraneters,-and-fotind- that-rro darrgerous- $€ € B-$ mirrimra could be-founct Is this the last word on CCB bounds? Hardly. - - - - One matst censider-that the paranneter space - we-studied-was vast; but by-no neans -exhaus - tive. Also, we studied only an universal model

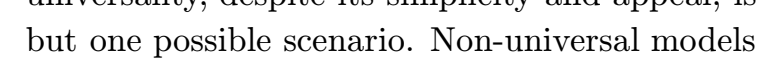
i could _possibly exhibit_dangerous_ CCB minima but notice that our arguments are not dependent on universality - again, the full minimisation conditions should be considered. We hope to have convinced the reader that great care must be ex' ercised_in_considering the problem_of_the_1-loمop. contributions to the potential in the case of $\mathrm{CCB}$ minima, and a re-evaluation of $\mathrm{CCB}$ bounds , is in order._Einally: we haye been trying_our_very - best-to-limit-the-study of CCB -to-the-tree-level potential, understandingly, given its simplicity. However, maybe we have reached a point where that is no longer possible - our strategy has been, choose a value of $\mu$ such that $V_{1}$ is minimal, and

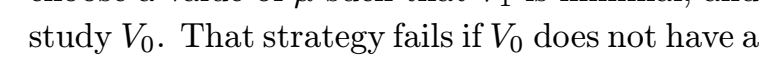
- minimum; and, as we saw, solving the minimisation equations can be very difficult. Perhaps then we should follow a different approach: choose $\mu$ such that the minimisation of $V_{0}$ is possible, thus ensuring the integrity of the 1-loop potential. If at this scale $V_{1}$ is not negligible, then we will have no alternative than to compute the 1-loop corrections to the vevs of the different fields. We hope to be able to explore this possibility soon.

\section{Acknowledgments}

I thank Giovanni Ridolfi and Tim Jones for very useful discussions. This work was supported by the European Commission TMR program ERB FMRX-CT96-0045.

\section{References}

[1] J.M. Frére, D.R.T. Jones and S. Raby, 'í Phys. B 222 (1983) 11'.

[2] L. Alvarez-Gaumé, J. Polchinski and M. Wise, Nucl. Phys. B 221 (1983) 495; J.P. Derendinger and C.A. Savoy Nucl. Phys. B 237 (1984) 307; C. Kounnas, A.B. Lahanas, D.V. Nanopoulos and M. Quirós, 'Nucl. Phys. B 236 (1984) 438'; M. Claudson, L.J. Hall and I. Hinchliffe, iNucl.' Phys. B 228 (1983) 501; M. Drees, M. Glück and K. Grassie, 'Phys. Lett. B 157. (1985) 164".

[3] J.F. Gunion, H.E. Haber and M. Sher, iNucl.' Phys._B_331_(1988) 320

[4] G. Gamberini,_G. Ridolfi and F. Zwirner, ínucl.' Phys. B.331 (1990) 331'

[5] J.A. Casas, A. Lleyda and C. Muñoz, 'Nucl.'s Phys. B_471_(1996)_3!

[6] U. Ellwanger and C. Hugonie, ihep-ph/9811386; S. Abel and T. Falk, 'Phys. Lett. B 444 (1998)! $4 \overline{2} \overline{7}_{p}$ S. Abel and C. Savoy, Phys. Lett. B $\mathbf{4} 4$ (1998) $1199_{i}^{\prime}$ S. Abel and B. Allanach, iPhys. Lett.' B 431 (1998) 339.

[7] Y. Fujimoto, L. O'Raifeartaigh and G. Parravicini, iNucl. Phys. B $\mathbf{2} \overline{2}(1983)$ 268i; $\mathrm{E}$. Weinberg and A. Wu, IPhys. Rev. D 36 (1987)! 2474 .

[8] C. Caso et al, Eur. Phys. Jour. C 3 (1998) 1.

[9] V. Barger, M.S. Berger and P. Ohmann, 'Phys.' Rev. D 49 (1994) 4908.

[10] M. Claudson, L.J. Hall and I. Hinchliffe, 'Nučl.' Phys. B 228 (1983) 501',

[11] P.M. Ferreira, under preparation. 